\title{
Assessment of Service Quality in Wireless Sensor Networks with Probabilistic Linguistic Term Sets
}

\author{
https://doi.org/10.3991/ijoe.v13i03.6865 \\ Pinghua Zhang* \\ Hunan College of Information, Changsha, China \\ Hunan University, Changsha, China \\ Zph_hncoi12@126.com \\ Shuangxiang She \\ Powerchina Zhongnan Engineering Co. Ltd, Changsha, China \\ lishijun 820404 @qq.com
}

\begin{abstract}
Wireless sensor networks as an emerging technology have garnered a lot of attention recently. With the development of wireless sensor networks, some problems such as data delay, information loss, and others have gradually appeared. In order to cope with these problems, evaluating the service quality in wireless sensor networks is crucial. However, how to evaluate the service quality in wireless sensor networks and especially how to accurately portray the preferences of a decision maker exactly are often difficult. To deal with this challenge, firstly, an evaluation system of attributes related to the service quality in wireless sensor networks is constructed based on the existing studies. Then, the probabilistic linguistic method including the definition of probabilistic linguistic term sets, the operators of probabilistic linguistic term sets, and the ranking order of probabilistic linguistic term sets are introduced. Probabilistic term sets used to denote the preferences of a decision maker are considered as more appropriate expressions than classical linguistic term sets, 2-tuple linguistic term sets, and portion linguistic term sets in the process of evaluating the service quality in wireless sensor networks. Finally, the probabilistic linguistic method is applied in the constructed service quality in wireless sensor networks so as to demonstrate its validity and applicability and further to help the decision maker find the problems in the service quality in wireless sensor networks and improve them.
\end{abstract}

Keywords-service quality; wireless sensor network; assessment; probabilistic linguistic term sets

\section{Introduction}

With the rapid development of computer technologies and communication technologies, embedded computing technology has been widely adopted and applied in many areas, especially wireless sensor networks [1-2]. As these technologies are mutual fusion, micro sensors which have perception, calculation, and communication 
abilities have been developed and have become the basis of wireless sensor networks. Wireless sensor networks combine the advantages of transducer and embedded technology with distributed information processing and those of communication technology. In general, wireless sensor networks have three nodes, namely the managed node, aggregated node, and sensor node [2-3]. Here, reliable, self-organizing, and multi-hop networks are used to monitor the operation of wireless sensor networks in real time and collect and address relative information in a special environment and transmit this information to users. Up to date, wireless sensor networks have been widely applied in national defense, agriculture and industry, urban management, as well as in biological, medical, and environmental monitoring [6-7].

Luan et al., (2015) summarized the characteristics of wireless sensor networks as follows, namely low cost, small volume, and self-organization and introduced a comprehensive evaluation method to evaluate the performance of wireless sensor networks [1]. Li (2009) analyzed the existing important models of wireless sensor networks, such as the sensor model, communication model, energy model, and so on. He further proposed three sub-optimal fusion rules to ensure the high quality of detection [2].

However, in the process of transmitting information, channel congestion, loss of data, and transmission delay may frequently occur. It is therefore necessary to develop a way to improve the service quality in wireless sensor networks. Thus, the first step is to introduce a method to evaluate the existing service quality in wireless sensor networks. Through this evaluation and analysis, the problems in the existing service quality in wireless sensor networks can be easily found, analyzed, and improved.

Li (2012) designed a wireless sensor network experiment platform, including hardware and software, to research and verify wireless sensor networks and the related technology of service. In order to improve the standard of service quality in wireless sensor networks and reduce the negative impact of inherent network time delay, Li proposed a new multi-objective optimization algorithm in his research [3]. Chen and $\mathrm{Li}$ (2010) introduced a routing algorithm for wireless sensor networks based on the geographical location of sensor nodes and energy consumption in order to fit the source node and effectively control the transmission path [4]. Finally, Wang and Yang (2012) proposed a new protocol specification of ZigBee protocol in wireless sensor networks to evaluate the service quality in wireless sensor networks [5].

In order to construct an evaluation system for the service quality in wireless sensor networks, in this paper, we introduce the fuzzy linguistic method. Linguistic variables such as very high, high, very low, low, and so on are introduced to help the decision maker or expert express their preferences about some alternatives on several attributes [8-9]. Here, in order to copy with different expressions of the decision makers, different forms of linguistic information are proposed, including classical linguistic information, intuitionistic fuzzy linguistic information, hesitant fuzzy linguistic information, dual-hesitant linguistic information, and so on [10-11]. However, these expressions cannot handle a special situation. For example, when a decision maker wants to express some linguistic variables simultaneously instead of a single linguistic variable, they are not available. Thus, probabilistic linguistic term sets were intro- 
duced by Pang et al., (2016) to express the preferences of the decision makers exactly and comprehensively [12].

In this paper, a new evaluation system for the service quality in wireless sensor networks has been constructed. Firstly, the relative concepts of service quality in wireless sensor networks are demonstrated and their meaning analyzed. Secondly, several attributes of the service quality in wireless sensor networks are selected based on the existing studies of other researchers. An attribute system is then constructed. Thirdly, some concepts related to probabilistic linguistic term sets, including the definitions of classical linguistic term sets and probabilistic linguistic term sets, are discussed. Then, operational laws and operators of probabilistic linguistic term sets are introduced. Owing to some drawbacks of the existing probabilistic linguistic term sets, a new, normalized way of probabilistic linguistic term sets has been developed in this paper to depict the probability information provided by the decision makers more exactly. Finally, based on the proposed probabilistic linguistic method, we evaluate the service quality in four wireless sensor networks and select the best one to help other networks improve their performance and determine their problems. This process also verifies the validity of the constructed evaluation system in the service quality in four wireless sensor networks.

The rest of this paper is organized as follows. The framework of the service quality in wireless sensor networks is constructed in Section 2. Section 3 introduces the proposed probabilistic linguistic method and its detailed algorithms. Section 4 demonstrates the application process of the proposed probabilistic linguistic method in the constructed framework of the service quality in four wireless sensor networks and analyzes its results to help the decision maker improve the service quality in four wireless sensor networks. A conclusion and references follow.

\section{Framework of service quality assessment in wireless sensor networks}

Research on wireless sensor networks dates back to that conducted by the military in the 1970s. Until 1995, this technology has gradually attracted a lot of attention from academic and practical fields. Wireless sensor networks integrate network technology, integrated circuits, wireless communication, and sensors with MEMS. Recently, the service quality in wireless sensor networks has been a hot topic in the academic field. Some factors in the problem of evaluating the service quality in wireless sensor networks should be considered. For example, wireless sensor networks should be regarded as a whole. The characteristics of nodes in wireless sensor networks should be analyzed and the corresponding agreement should be also considered. Based on the existing studies, several attributes are selected in Figure 2 below. 


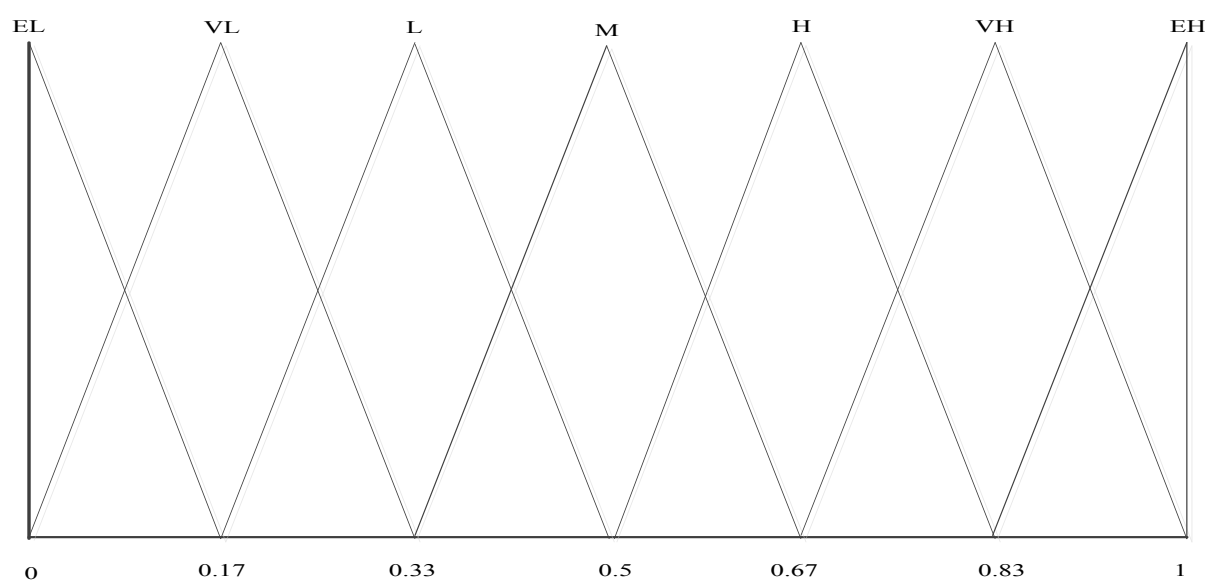

Fig. 1. Demonstration of a set of linguistic terms

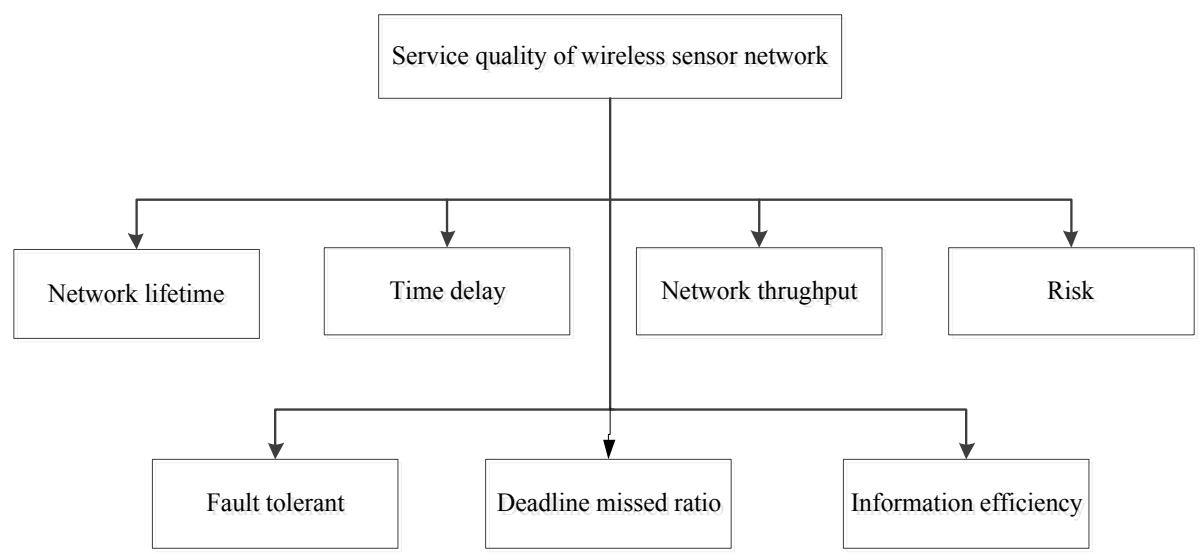

Fig. 2. System of attributes in the evaluation problem

\section{$3 \quad$ Probabilistic linguistic term sets}

In general, when a decision problem includes not only quantitative information but also qualitative information, qualitative information is more important, and linguistic term sets are considered an appropriate method to help experts express their preferences about several alternatives as analyzed in the Introduction. Information in the linguistic approach is denoted by means of linguistic variables.

\subsection{Basic concepts related to linguistic term sets}

Before the linguistic approach is used, the linguistic descriptors should be firstly provided, including several orders of linguistic terms. For example, a set of seven terms $T$ can be given as follows. 
$T=\left\{t_{0}=E L, t_{1}=V L, t_{2}=L, t_{4}=M, t_{5}=H, t_{6}=V H, t_{7}=E H\right\}$,

Where $N$ denotes Extreme Low, $V L$ denotes Very Low, $L$ denotes Low, $M$ denotes Medium, $H$ denotes High, $V H$ denotes Very High, and $P$ denotes Extreme High. This is demonstrated in Figure 1.

Given a linguistic scale or linguistic term set $T=\left\{t_{0}, \ldots, t_{i}, \ldots, t_{g}\right\}$, experts can employ any linguistic term to express their preferences towards alternatives in decision making. The classic linguistic term set should satisfy the following operators (Herrera et al. 2000).

1. A negation operator: $\operatorname{Neg}\left(t_{i}\right)=t_{j}$ such as $j=g+1-i(g+1$ is called the cardinality or granularity of the linguistic term set).

2. An order of $T: t_{i}<t_{j}$, if and only if $i<j$.

3. A max operator: $\max \left(t_{i}, t_{j}\right)=t_{i}$, if and only if $t_{i}>t_{j}$.

4. A min operator: $\min \left(t_{i}, t_{j}\right)=t_{i}$, if and only if $t_{i}<t_{j}$.

The mentioned classic linguistic term sets are discrete. The fact that aggregated linguistic terms cannot be denoted by discrete linguistic term sets directly may lead to difficult operation processes. Xu (2004) and Herrera et al. (2000) developed the existing discrete linguistic term set $T$ and proposed the continuous linguistic term set $T=$ $\left\{t_{q} \mid q \in[-p, p]\right\}$. Here, parameter $p(p>g)$ represents a sufficiently large positive number. It is obvious that the aggregated linguistic term may be a continuous linguistic term which is named virtual linguistic term.

Therefore, the operational laws of linguistic term sets can be further defined as follows.

(1) $t_{i} \oplus t_{j}=t_{j} \oplus t_{i}=t_{i+j}$,

(2) $t_{i} \oplus t_{j}=t_{i+j}$,

(3) $\beta t_{i}=t_{\beta i}$,

(4) $\beta\left(t_{i} \oplus t_{j}\right)=\beta t_{i} \oplus \beta t_{j}, \beta \in[-1,1]$.

These operational laws should be applied to generate aggregation operators so as to help the decision maker aggregate linguistic decision information.

\subsection{Basic concepts of probabilistic linguistic term sets}

As mentioned in the Introduction, with the increasing complexity of decision problems, a single linguistic term cannot exactly describe experts' preferences. It is necessary to develop probability linguistic term sets in order to portray decision information provided by experts more flexibly, exactly, and comprehensively. That means that several linguistic terms could express experts' preferences simultaneously. In particular, ignorance or uncertainty can be described in this expression. It is assumed that if the expert does not provide partial decision information, the information should be considered in this expression. This idea has been supported by the evidential reasoning method proposed by Yang and Xu (2002) [11]. However, the grades in the evidential reasoning method are not aggregated directly. The utility theory was intro- 
duced in the evidential reasoning to indicate the value of each grade. However, in linguistic decision making, the subscript instead of the utility represents the value of each linguistic term. This way is clear and explicit for the decision makers to realize the meaning of linguistic terms when they use them. In general, the decision makers cannot provide their preferences by using a single linguistic term but need to use several linguistic terms simultaneously as mentioned earlier. Thus, probabilistic linguistic term sets as an appropriate way have been developed by Pang et al. (2016) [12]. Pang et al., (2016) stated that the decision makers may be hesitant about selecting from several possible linguistic terms, especially when they want to express their preferences exactly towards some alternatives. Then, the definition of probabilistic linguistic term sets is demonstrated as follows.

Definition 1. Suppose $T=\left\{t_{0}, t_{1}, \ldots, t_{g}\right\}$ represents a linguistic term set. The probabilistic linguistic term set is defined as

$$
\mathrm{P}=\left\{\left(t_{x}, p_{x}\right) \mid t_{x} \in T, p_{x} \geq 0, x=1,2, \ldots, \#\left(t_{x}\right), \sum_{x=1}^{\#\left(t_{x}\right)} p_{x} \leq 1\right\}
$$

Here, $\left(t_{x}, p_{x}\right)$ as a binary variable denotes a linguistic term $t_{x}$ and its probability $p_{x}$ provided by the decision makers. $\#\left(t_{x}\right)$ represents the number of the linguistic terms in a linguistic term set. It should be noted that when the sum of probability of all linguistic terms is equal to 1 denoted by $\sum_{x=1}^{\#\left(t_{x}\right)} p_{x}=1$, this linguistic term set is considered as complete. Or else, it is considered as incomplete when the sum probability of all linguistic terms provided by the decision makers $\sum_{x=1}^{\#\left(t_{x}\right)} p_{x}<1$. That means that partial information is unknown. The decision makers do not provide all preferences and they have some hesitancy over decision alternatives due to their limited knowledge and work experience. This phenomenon may often occur in real cases, especially in evaluating the service quality in wireless sensor networks. In particular, when the sum of probability of all linguistic terms is equal to 0 denoted by $\sum_{x=1}^{\#\left(t_{x}\right)} p_{x}=0$, the preferences of the decision makers towards decision alternatives are completely unknown. It is an extreme situation in real cases. Thus, we give an example to demonstrate this concept below.

Example 1. Given a linguistic term set denoted by $T=\left\{t_{0}, t_{1}, \ldots, t_{7}\right\}$, a decision maker gives his preferences towards three cars of the brands Geely, Hyundai, and Ford by using probabilistic linguistic term sets as follows.

$$
\begin{aligned}
& P_{1}=\left\{\left(t_{4}, 0.8\right),\left(t_{5}, 0.2\right)\right\}, \\
& P_{2}=\left\{\left(t_{4}, 0.5\right),\left(t_{5}, 0.4\right)\right\}, \\
& P_{3}=\left\{\left(t_{4}, 0.4\right),\left(t_{5}, 0.4\right),\left(t_{6}, 0.1\right)\right\} .
\end{aligned}
$$




\subsection{Information aggregation}

Before decision information is aggregated, more importantly, how to address ignorance information in a linguistic term set is a challenge. Pang et al. (2016) proposed a new way to normalize probabilistic linguistic term sets.

Definition 2. Suppose $T=\left\{t_{0}, t_{1}, \ldots, t_{g}\right\}$ represents a linguistic term set. The probabilistic linguistic term set is denoted by $P=\left\{\left(t_{x}, p_{x}\right) \mid x=1,2, \ldots, \#\left(t_{x}\right)\right\}$ and $\sum_{x=1}^{\#\left(t_{x}\right)} p_{x}<1$. Then, the normalized probabilistic linguistic term set is defined as

$$
\beta / \#\left\{\left(t_{x}, \beta 0\right) \mid x=1,2, \ldots, \#\left(t_{x}\right)\right\}
$$

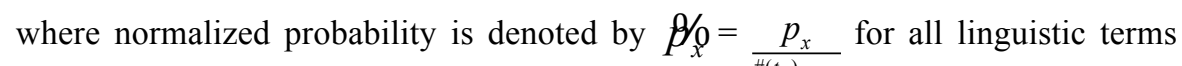
denoted by $x=1,2, \ldots, \#\left(t_{x}\right)$.

From Definition 2, it is obvious that normalized probabilistic term sets include probability information which removes incomplete probability information. In other words, unknown information is averagely divided into the known linguistic terms. However, there is no additional information provided by the decision makers to express this preference. Thus, this way to handle ignorance information in a linguistic term set is not very appropriate. In particular, some special situations cannot be explained well. For example, when a decision maker gives a preference denoted by $P=$ $\left\{\left(t_{2}, 0.8\right)\right\}$ using a probabilistic linguistic term set, the normalized probabilistic linguistic term obtained is $\beta / 0=\left\{\left(t_{2}, 1\right)\right\}$. Obviously, it is inconsistent. This normalized probabilistic linguistic term does not show the exact preferences of the decision maker. So, this way of normalizing incomplete linguistic term sets provided by Pang et al. (2016) is not reasonable. In order to overcome this difficulty, we propose an idea. The unknown information in a probabilistic linguistic term set should not be removed and be combined in the aggregation process.

Through the above analysis, the aggregation operator of probabilistic linguistic term sets is proposed in the following.

Definition 3. Suppose $T=\left\{t_{0}, t_{1}, \ldots, t_{g}\right\}$ represents a linguistic term set and the probabilistic linguistic term set is denoted by $P_{i}=\left\{\left(t_{x, i}, p_{x, i}\right) \mid x=1,2, \ldots, \#\left(t_{x, i}\right)\right\}$ and $\sum_{x=1}^{\#\left(t_{x, i}\right)} p_{x, i}<1$. Then, the probabilistic linguistic averaging operator is defined as

$$
P A\left(P_{1}, \ldots, P_{i}, \ldots, P_{n}\right)=\frac{1}{n}\left(P_{1} \oplus \ldots \oplus P_{i} \oplus \ldots \oplus P_{n}\right)
$$




$$
=\frac{1}{n}\left\{t_{x, 1} \cdot p_{x, 1} \oplus \ldots \oplus t_{x, i} \cdot p_{x, i} \oplus \ldots \oplus t_{x, n} \cdot p_{x, n}\right\} .
$$

Definition 4. Suppose $T=\left\{t_{0}, t_{1}, \ldots, t_{g}\right\}$ represents a linguistic term set and the probabilistic linguistic term set is denoted by $P_{i}=\left\{\left(t_{x, i}, p_{x, i}\right) \mid x=1,2, \ldots, \#\left(t_{x, i}\right)\right\}$ and $\sum_{x=1}^{\#\left(t_{x, i}\right)} p_{x, i}<1$, and attribute weight can be obtained and denoted by $w=w_{1}, \ldots, w_{i}, \ldots w_{n}$ and $\sum_{i=1}^{n} w_{i}=1$. Then, the probabilistic linguistic weighted averaging operator is defined as

$$
\begin{array}{r}
P W A\left(P_{1}, \ldots, P_{i}, \ldots, P_{n}\right)=\left(w_{1} P_{1} \oplus \ldots \oplus w_{i} P_{i} \oplus \ldots \oplus w_{n} P_{n}\right) \\
=\left\{w_{1} t_{x, 1} \cdot p_{x, 1} \oplus \ldots \oplus w_{i} t_{x, i} \cdot p_{x, i} \oplus \ldots \oplus w_{n} t_{x, n} \cdot p_{x, n}\right\}
\end{array}
$$

Definition 5. Suppose $T=\left\{t_{0}, t_{1}, \ldots, t_{g}\right\}$ represents a linguistic term set and the probabilistic linguistic term set is denoted by $P_{i}=\left\{\left(t_{x, i}, p_{x, i}\right) \mid x=1,2, \ldots, \#\left(t_{x, i}\right)\right\}$ and $\sum_{x=1}^{\#\left(t_{x, i}\right)} p_{x, i}<1$. Then, the probabilistic linguistic geometric operator is defined as

$$
\begin{aligned}
& P G\left(P_{1}, \ldots, P_{i}, \ldots, P_{n}\right) \\
& =\left(\left(P_{1}\right) \otimes \ldots \otimes\left(P_{i}\right) \otimes \ldots \otimes\left(P_{n}\right)\right)^{1 / n} \\
& \quad\left\{\left(p_{x, 1}\right)^{t_{x, 1}} \otimes \ldots \otimes\left(p_{x, i}\right)^{t_{x, i}} \otimes \ldots \otimes\left(p_{x, n}\right)^{t_{x, n}}\right\}^{1 / n} .
\end{aligned}
$$

Definition 6. Suppose $T=\left\{t_{0}, t_{1}, \ldots, t_{g}\right\}$ represents a linguistic term set and the probabilistic linguistic term set is denoted by $P_{i}=\left\{\left(t_{x, i}, p_{x, i}\right) \mid x=1,2, \ldots, \#\left(t_{x, i}\right)\right\}$ and $\sum_{x=1}^{\#\left(t_{x, i}\right)} p_{x, i}<1$, and attribute weight can be obtained and denoted by $w=w_{1}, \ldots, w_{i}, \ldots w_{n}$ and $\sum_{i=1}^{n} w_{i}=1$. Then, the probabilistic linguistic weighted geometric operator is defined as

$$
\begin{aligned}
& P W G\left(P_{1}, \ldots, P_{i}, \ldots, P_{n}\right)= \\
& \left(\left(P_{1}\right)^{w_{i}} \otimes \ldots \otimes\left(P_{i}\right)^{w_{i}} \otimes \ldots \otimes\left(P_{n}\right)^{w_{n}}\right)
\end{aligned}
$$




$$
=\left\{\left(p_{x, 1}\right)^{w_{i} t_{x, 1}} \otimes \ldots \otimes\left(p_{x, i}\right)^{w_{i} t_{x, i}} \otimes \ldots \otimes\left(p_{x, n}\right)^{w_{n} t_{x, n}}\right\} .
$$

\subsection{Ranking alternatives}

After the probabilistic linguistic information is aggregated, the next problem is the ranking order of the alternatives. Because a probabilistic linguistic term set as a distribution includes several linguistic terms, two probabilistic linguistic term sets cannot be compared with each other directly. Then, the score of probabilistic linguistic term sets is introduced in the following.

Definition 7. Suppose $T=\left\{t_{0}, t_{1}, \ldots, t_{g}\right\}$ represents a linguistic term set and the probabilistic linguistic term set is denoted by $P=\left\{\left(t_{x}, p_{x}\right) \mid x=1,2, \ldots, \#\left(t_{x}\right)\right\}$ and $\sum_{x=1}^{\#\left(t_{x}\right)} p_{x}<1$. Then the score of the probabilistic linguistic term set $P_{i}$ is defined as

$$
\mathrm{V}(\mathrm{P})=\frac{\sum_{x=1}^{\#\left(t_{x}\right)} t_{x} \cdot p_{x}}{\sum_{x=1}^{\#\left(t_{x}\right)} p_{x}}
$$

Thus, for two probabilistic linguistic term sets $P_{1}$ and $P_{2}$,

(1) if $V\left(P_{1}\right)>V\left(P_{2}\right), P_{1}>P_{2}$;

(2) if $V\left(P_{1}\right)<V\left(P_{2}\right), P_{1}<P_{2}$;

(3) if $V\left(P_{1}\right)=V\left(P_{2}\right), P_{1}=P_{2}$.

\section{The procedure of service quality assessment}

As demonstrated in Section 2, the attribute system of service quality in wireless sensor networks is constructed. Then, the problem of evaluating the service quality in wireless sensor networks is described in the following.

There are four wireless sensor networks which are considered as alternatives in the proposed decision making method and are denoted as $A_{1}, A_{2}, A_{3}, A_{4}$. The set of attributes which is applied to assess the four alternatives is denoted as $C_{1}, C_{2}, C_{3}, C_{4}, C_{5}, C_{6}, C_{7}$, and the weights for these attributes are provided by the decision maker and are denoted as $w=\{0.1,0.15,0.1,0.25,0.1,0.2,0.1\}$ which is generated based on the work experience and background of the decision maker related to the wireless sensor networks. A set of seven terms $T$ is also provided by the decision maker as $T=\left\{t_{0}=E L, t_{1}=V L, t_{2}=L, t_{3}=M, t_{4}=H, t_{5}=V H, t_{6}=E H\right\}$. He or she will express his or her preferences towards the four alternatives on seven attributes by using this linguistic term set.

Then, the decision maker provides assessments by using the probabilistic linguistic terms based on his or her preferences for each alternative $\mathrm{A}_{i}(i=1,2, \ldots, \mathrm{s})$ with re- 
spect to each attribute $C_{j}(j=1,2, \ldots, 7)$. The decision matrix is obtained and illustrated in Table 1. After that, the probabilistic linguistic weighted averaging operator mentioned in Eq. (4) is introduced to combine the assessments of each attribute. The ranking order is generated by using Eq. (7) and based on Definition 7, which is denoted by $A_{2}>A_{4}>A_{3}>A_{1}$. So, the result of this evaluation problem is obtained and $\mathrm{A}_{i}$ is considered as the best in this problem. The differences between these networks will be further discussed in future research.

Table 1. Transposition of the DHF decision matrix

\begin{tabular}{|l|l|l|l|l|}
\hline & \multicolumn{1}{|c|}{$\boldsymbol{A}_{1}$} & \multicolumn{1}{|c|}{$\boldsymbol{A}_{2}$} & \multicolumn{1}{c|}{$\boldsymbol{A}_{3}$} & \multicolumn{1}{c|}{$\boldsymbol{A}_{4}$} \\
\hline$C_{1}$ & $\left(\left\{t_{1}, 0.5\right\},\left\{t_{2}, 0.5\right\}\right)$ & $\left(\left\{t_{4}, 0.4\right\},\left\{t_{5}, 0.6\right\}\right)$ & $\left(\left\{t_{5}, 1\right\}\right)$ & $\left(\left\{t_{3}, 0.7\right\},\left\{t_{4}, 0.3\right\}\right)$ \\
\hline$C_{2}$ & $\left(\left\{t_{4}, 0.3\right\},\left\{t_{5}, 0.7\right\}\right)$ & $\left(\left\{t_{2}, 0.5\right\},\left\{t_{3}, 0.5\right\}\right)$ & $\begin{array}{l}\left(\left\{t_{4}, 0.3\right\},\left\{t_{5}, 0.2\right\},\left\{t_{6},\right.\right. \\
0.4\})\end{array}$ & $\left(\left\{t_{2}, 0.3\right\},\left\{t_{5}, 0.6\right\}\right)$ \\
\hline$C_{3}$ & $\left(\left\{t_{3}, 0.2\right\},\left\{t_{4}, 0.8\right\}\right)$ & $\left(\left\{t_{5}, 0.7\right\},\left\{t_{3}, 0.3\right\}\right)$ & $\left(\left\{t_{3}, 0.4\right\},\left\{t_{4}, 0.4\right\}\right)$ & $\left(\left\{t_{3}, 0.4\right\},\left\{t_{4}, 0.5\right\}\right)$ \\
\hline$C_{4}$ & $\left(\left\{t_{5}, 0.9\right\}\right)$ & $\left(\left\{t_{5}, 0.2\right\},\left\{t_{6}, 0.8\right\}\right)$ & $\left(\left\{t_{3}, 0.5\right\},\left\{t_{5}, 0.4\right\}\right)$ & $\left(\left\{t_{3}, 0.6\right\},\left\{t_{4}, 0.3\right\}\right)$ \\
\hline$C_{5}$ & $\left(\left\{t_{3}, 1\right\}\right)$ & $\left(\left\{t_{3}, 0.2\right\},\left\{t_{4}, 0.6\right\}\right)$ & $\left(\left\{t_{1}, 0.4\right\},\left\{t_{2}, 0.5\right\}\right)$ & $\begin{array}{l}\left(\left\{t_{2}, 0.3\right\},\left\{t_{3}, 0.3\right\},\left\{t_{4},\right.\right. \\
0.3\})\end{array}$ \\
\hline$C_{6}$ & $\left(\left\{t_{2}, 0.5\right\},\left\{t_{3}, 0.3\right\},\left\{t_{4}, 0.2\right\}\right)$ & $\left(\left\{t_{3}, 0.2\right\},\left\{t_{5}, 0.5\right\}\right)$ & $\left(\left\{t_{3}, 0.8\right\}\right)$ & $\left(\left\{t_{1}, 0.8\right\},\left\{t_{2}, 0.2\right\}\right)$ \\
\hline$C_{7}$ & $\left(\left\{t_{1}, 0.4\right\},\left\{t_{3}, 0.5\right\}\right)$ & $\left(\left\{t_{4}, 1\right\}\right)$ & $\left(\left\{t_{2}, 0.4\right\},\left\{t_{3}, 0.5\right\}\right)$ & $\begin{array}{l}\left(\left\{t_{1}, 0.5\right\},\left\{t_{2}, 0.4\right\},\left\{t_{3},\right.\right. \\
0.1\})\end{array}$ \\
\hline
\end{tabular}

\section{Conclusions}

This paper has proposed a method to evaluate the service quality in wireless sensor networks, which are considered as an emerging technology and have attracted a lot of attention from the academic and practical fields. Some problems such as data delay, information loss, and others have been found in wireless sensor networks recently. In order to cope with these problems, firstly, an evaluation system of attributes related to the service quality in wireless sensor networks is constructed based on the existing studies. Then, the probabilistic linguistic method including the definition of probabilistic linguistic term sets, the operators of probabilistic linguistic term sets, and ranking order of probabilistic linguistic term sets is introduced. Probabilistic term sets used to denote the preferences of a decision maker are considered more appropriate than other expressions such as classical linguistic term sets, 2-tuple linguistic term sets, and portion linguistic term sets in the process of evaluating the service quality in wireless sensor networks. Finally, the probabilistic linguistic method is applied in the constructed service quality in wireless sensor networks so as to demonstrate its validity and applicability and further help the decision maker find the problems in the service quality in wireless sensor networks and improve them.

In the future, the result of this evaluation problem will be further analyzed and improved. In addition, this method may be applied in wider areas in wireless sensor networks. 


\section{References}

[1] Luan, J., Wang, Q., He, X., Guan, F., Bian, E., He, J. (2015). Analysis of wireless sensor network performance evaluation. Machine Building and Automation, 44(3), 165-167.

[2] Lin, J. (2009). Quality of service support for event detection in wireless sensor networks. Zhejiang University Press.

[3] Lin, J. (2012). Analysis and experimental research on QOS management problem of wireless sensor network, China Jiliang University Press.

[4] Cheng, Z., Li, L. (2010). A QOS routing algorithm based on geographical location information for wireless sensor networks, Journal of Shanghai Jiao Tong University, 44(3), 413-417.

[5] Wang, J. G., \& Yang, J. (2012). Research for wireless sensor networks quality of service on the zigbee protocol. Electronic Design Engineering. 20(22), 138-140.

[6] Chanak, P., Banerjee, I., \& Sherratt, R. S. (2016). Energy-aware distributed routing algorithm to tolerate network failure in wireless sensor networks. Ad Hoc Networks. 56(1), 158-172.

[7] Sahoo, P. K., \& Sheu, J. P. (2017). Design and analysis of collision free mac for wireless sensor networks with or without data retransmission. Journal of Network \& Computer Applications, 80, 10-21. https://doi.org/10.1016/j.jnca.2016.12.020

[8] Herrera, F., \& Herrera-Viedma, E. (2000). Linguistic decision analysis: steps for solving decision problems under linguistic information. Fuzzy Sets \& Systems, 115(1), 67-82. https://doi.org/10.1016/S0165-0114(99)00024-X

[9] Herrera, F., \& Martinez, L. (2000). A 2-tuple fuzzy linguistic representation model for computing with words. IEEE Transactions on Fuzzy Systems, 8(6), 746-752. https://doi.org/10.1109/91.890332

[10] Xu, Z. (2004). Uncertain multiple attribute decision making methods and applications. Tsinghua University Press, Beijing.

[11] Yang, J. B., \& Xu, D. L. (2002). On the evidential reasoning algorithm for multiple attribute decision analysis under uncertainty. Systems Man \& Cybernetics Part A Systems \& Humans IEEE Transactions on, 32(3), 289-304. https://doi.org/10.1109/TSMCA. 2002.802746

[12] Pang, Q., Wang, H., \& Xu, Z. (2016). Probabilistic linguistic term sets in multi-attribute group decision making. Information Sciences, 369, 128-143. https://doi.org/10.1016/ j.ins.2016.06.021

\section{$7 \quad$ Authors}

Pinghua Zhang (corresponding author), is an associate professor in the school of Electronic Engineering, Hunan College of Information, 410200, Changsha, China. She received a Master degree from Hunan Normal University. Her current research areas are wireless communications and signal processing. She is also affiliated with College of Computer Science and Electronic Engineering, Hunan University, 410082, Changsha, China (Zph_hncoi12@126.com).

Shuangxiang She is a master of Powerchina Zhongnan Engineering Co. Ltd., Changsha 410014, China. He is mainly engaged in communication technology research (lishijun820404@qq.com).

Article submitted 23 February 2017. Published as resubmitted by the authors 25 March 2017 